\title{
IN VITRO APPROACH FOR IDENTIFICATION OF A LEADING CYTOCHROME P450 ISOENZYME RESPONSIBLE FOR BIOTRANSFORMATION OF NOVEL ARYLPIPERAZINE DRUG CANDIDATES AND THEIR INHIBITION POTENCY TOWARDS CYP3A4
}

\author{
SZYMON ULENBERG ${ }^{1}$, MARIUSZ BELKA ${ }^{1}$, PAWEŁ GEORGIEV ${ }^{1}$, MAREK KRÓL² \\ FRANCISZEK HEROLD ${ }^{2}$ and TOMASZ BĄCZEK ${ }^{1 *}$
}

\author{
${ }^{1}$ Department of Pharmaceutical Chemistry, Medical University of Gdańsk, Poland \\ ${ }^{2}$ Department of Drug Technology and Pharmaceutical Biotechnology, \\ Medical University of Warsaw, Poland
}

\begin{abstract}
The aim of this study was to identify cytochrome P450 (CYP) isoforms that participate in the metabolism of some novel arylpiperazine derivatives developed by authors as well as their potency to inhibit reactions catalyzed by identified lead metabolizing enzyme. Such studies allow to predicting possible drug-drug interactions that might occur during co-administration of studied compounds with other drugs that are metabolized by an identified enzyme. The compounds were incubated in vitro together with the isolated CYP isoforms. After the incubation, samples were analyzed by liquid chromatography coupled with mass spectrometry. The results showed the main contribution of CYP3A4 isoform in biotransformation of the investigated derivatives. With CYP3A4 being the main CYP isoform responsible for the metabolism of arylpiperazine derivatives and at the same time being the main metabolizing enzyme for almost $50 \%$ of all drugs, a high chance of in vivo drug-drug interactions emerged. Therefore, $\mathrm{IC}_{50}$ values were also determined using testosterone hydroxylation as a probe reaction, specific for CYP3A4. The resulting values ranged from 6.13 to $15.85 \mu \mathrm{M}$, which places studied derivatives as moderate or weak inhibitors of CYP3A4. Those results, combined with the conclusion that all of the arylpiperazine derivatives are also metabolized to some extent by other CYP isoforms (providing alternative metabolic pathways), result in a conclusion that studied arylpiperazines might be safe for co-administration with other CYP3A4 substrates.
\end{abstract}

Keywords: arylpiperazine, CYP 3A4 inhibitor, cytochrome P-450, isoform, metabolic stability, drug-drug interactions

Depressive disorder is a common mental illness, affecting approximately 300 million people worldwide. At its worst, depression can lead to suicide, with close to 800000 people committing suicide every year. Additionally, suicide is the second leading cause of death among 15- to 29-year-olds (1). Those numbers demonstrate a great need for new antidepressant agents, characterized by better efficacy and safety of use. Selective serotonin reuptake inhibitors (SSRIs) are one of the most popular groups of drugs for treating depression (2). Many studies have proved SSRIs efficacy in the treatment of mild and moderate depression $(3,4)$. One of their major flaws, however, is a long onset of clinical effect, which can take as long as 3-4 weeks. Studies by Artigas et. al. show that co-administration of paroxetine, a 5-HT $\mathrm{T}_{1 \mathrm{~A}}$ partial antagonist, together with pindolol, a $\beta$-adrenergic receptor antagonist, can reduce the onset time of anti-depressive action from 2-3 weeks to 3-7 days (5). When considering other 5-HT receptor ligands, arylpiperazine derivatives (Fig. 1) are a confirmed group of substances that have mostly $5-\mathrm{HT}_{1 \mathrm{~A}}$ and/or $5-\mathrm{HT}_{2 \mathrm{~A}}$ affinity. Being a relatively new class of psychotherapeutic drugs and drug candidates, they are partial agonists, showing a high affinity towards a $5-\mathrm{HT}_{1 \mathrm{~A}}$ receptor binding site, yet low selectivity for this receptor subtype. A model drug from this group, buspirone, along with its derivative trazodone, has gained wide acceptance for treating psychotic disorders accompanied by anxiety. All of the abovementioned properties make arylpiperazines worthy candidates for

\footnotetext{
* Corresponding author: e-mail: tbaczek@gumed.edu.pl
} 
combination treatment along with SSRIs, designed not only to decrease the time of clinical anti-depressive effects onset but also to help patients withstand the hardships of therapy and related stressors.

Large interest in arylpiperazines and their study is clearly seen in the literature. Considering the last six years, a significant number of novel derivatives have been synthesized (6-12). In the cited publications, in addition to new chemical entities, results of the structure-activity relationships have also been presented $(6,7,9-11)$. A study to model metabolic stability of arylpiperazines has also been conducted (13), not only describing an approach that can be applied to other compounds (14-17) but also identifying the best chemometric technique to conduct such research (18).

Together with the increasing depressive disorders incidence rate, there is also an increasing rate of comorbidities. This leads to multiple-drug therapies, thereby introducing the risk of interactions between different therapeutic agents. Many of the drug-drug interactions are based on hepatic CYP enzymes. Even though CYP has several isoforms capable of biotransformation of different types of drugs, CYPbased drug-drug interactions are among the most frequent in multi-drug therapy $(19,20)$.

Publications regarding buspirone confirm the leading contribution of CYP3A4 in its metabolism (21), which is worth noticing given its structural analogy with studied compounds. CYP3A4 is also a main metabolizing enzyme for statins, drugs used to treat hyperlipidemia, which - as the statistics show - is also a disorder with increasing incidence rate (22). Interactions occurring between statins and selective serotonin reuptake inhibitors have already been studied and proven to exist (23), showing the<smiles>[R]c1ccc(-c2c(=O)n(CCCCN3CCN([R7])CC3)c(=O)n3ccccc23)c([R])c1</smiles>

B<smiles>[R]c1ccc(-c2c3n(c(=O)n(CCCCN4CCN([R7])CC4)c2=O)CCCC3)c([R])c1</smiles>

\begin{tabular}{c|c|c|c} 
Compound & $\mathrm{R}$ & $\mathrm{R}^{\prime}$ & $\mathrm{R} "$ \\
\hline 1 & $\mathrm{CH}_{3}$ & $\mathrm{H}$ & 3- $\mathrm{CF}_{3}-\mathrm{Ph}$ \\
2 & $\mathrm{H}$ & $\mathrm{H}$ & 2-pyridyl \\
3 & $\mathrm{OCH}_{3}$ & $\mathrm{H}$ & 2-pyrimidinyl \\
4 & $\mathrm{~F}$ & $\mathrm{H}$ & 2-pyridyl \\
5 & $\mathrm{H}$ & $\mathrm{OCH}_{3}$ & 2-pyridyl
\end{tabular}

Figure 1. Detailed structure of the studied compounds. Structure A relates to compounds $\mathbf{1 - 4}$, structure B to compound $\mathbf{5}$. 
importance of the dangers of multiple-drug therapy. As statins are a very popular group of drugs, the possibility of them interacting with other drugs metabolized by CYP3A4 cannot be neglected. Therefore, studying the metabolic pathways of arylpiperazines (CYP3A4-metabolized buspirone derivatives) with emphasis on their inhibition potential towards various CYP isoforms in vitro might provide very valuable information regarding drug-drug interactions in which they may be involved. Along with the identification of the CYP isoform with leading contribution rate, this study also presents alternative metabolic pathways involving other CYP isoforms that greatly contribute to the safety of multi-drug therapy (by providing alternative ways for biotransformation in case of inhibition of the leading CYP isoform).

\section{EXPERIMENTAL}

\section{Studied compounds}

All studied arylpiperazines were synthesized according to the procedure presented in the previous study (24). Chemical structures of all 5 compounds (numbered 1-5) have been presented in Figure 1. The activity of derivatives towards $5-\mathrm{HT}_{1 \mathrm{~A}}, 5-\mathrm{HT}_{2 \mathrm{~A}}$ as well as $\alpha_{1}$ were also described and discussed in the same paper.

\section{Chemicals}

Incubations for determination of leading metabolizing CYP were conducted using P450 isolated isoenzymes in the form of cytochrome P450 BACULOSOMES $^{\circledast}$ Plus Reagents (Thermo Fisher Scientific, USA) with the addition of sodium salt of NADPH (Sigma-Aldrich, USA), monopotassium phosphate and dipotassium phosphate $(\mathrm{POCH}$, Poland), and deionized water (18 $\mathrm{M} \Omega$, MerckMillipore, Germany). Dimethyl sulfoxide (SigmaAldrich, USA) was used to prepare stock solutions of the studied compounds.

Incubations for $\mathrm{IC}_{50}$ value estimation were conducted using pooled human liver microsomes (Sigma-Aldrich, St. Louis, MO, USA). Testosterone, as well as 6-OH-testosterone, were purchased from Sigma-Aldrich, USA.

The mobile phase of the LC separation consisted of deionized water and Hypergrade LC-MS acetonitrile (Merck, Germany). Both mobile phases were prepared with the addition of ammonium formate buffer, which consisted of LC-MS formic acid and ammonium formate. The LC-MS internal standard was a $5 \mu \mathrm{M}$ buspirone hydrochloride solution.

\section{Contribution of different CYP450 isoenzymes in metabolism of the studied compounds}

Studied compounds were dissolved in dimethylsulfoxide and diluted in the phosphate buffer $(\mathrm{pH}=7.4)$ to obtain $100 \mu \mathrm{M}$ solutions. A 10 mM NADPH stock solution in the phosphate buffer was prepared ex tempore before each series of incubations. The final concentrations in the incubation mixes were $5 \mu \mathrm{M}$ of a studied substance, $0.5 \mathrm{nM}$ of a specific CYP (used CYPs were CYP1A2, CYP2A6, CYP2A9, CYP2C19, CYP2D6, CYP2E1, and CYP3A4) and, $100 \mu \mathrm{M}$ of NADPH in phosphate buffer ( $\mathrm{pH} 7.4,100 \mathrm{mM}$ ). The total volume of the reaction was $100 \mu \mathrm{L}$. Separate compounds were dosed into Microtiter 96-well plates. Pre-incubation mixes (a specific CYP with NADPH in phosphate buffer solution) were subjected for $5 \mathrm{~min}$ to the preincubation process under temperature of $37^{\circ} \mathrm{C}$. The main reaction was initiated afterward by adding a solution of the studied compound to the wells containing pre-incubation mixes. Incubations were conducted in an incubator, using a shaker and microtiter 96 well plates. To collect samples and determine the loss of compounds during incubation with a specific isoform, two sample-collection time points had to be designated. Those points were $t=0$ min (at the beginning of the reaction, to quantify the initial concentration of a compound in incubation mix) and $t=30 \mathrm{~min}$ (at the end of the reaction, to quantify the remaining compound in an incubation mix). To stop the reaction, ice-cold acetonitrile containing $5 \mu \mathrm{M}$ buspirone (as an internal standard) was used. Such a solution acts as a reaction-stopping reagent (due to its low temperature and acetonitrile for enzymes denaturation), and as an internal standard. After stopping the reaction, samples were centrifuged $(10 \mathrm{~min}, 7500 \mathrm{xg})$ to separate protein residue from the sample solution. Supernatants were directly analyzed using LC-MS.

Each incubation was repeated four times. To ensure both the validity of biological conditions and that no compound degradation related to the addition of enzyme solution occurs, a negative control was also included. Compositions of the negative compound mixes were the same as those used in the experiment with the exclusion of NADPH. Lack of NADPH (a cofactor for enzymatic reaction) causes CYP to lose their enzymatic activity; therefore, after incubation is performed, concentrations of the compounds in negative controls should not change significantly. Presented methodology uses baculosomes as an enzymatic system in the same concentrations. This situation does not fully reflect in vivo situation, as different CYP iso- 
forms have variable activity in the liver. For the purpose of this study, authors decided to use the same concentrations, however, this is a limitation of this methodology and a potential for future upgrade.

Influence of the studied compounds on CYP3A4 activity and designation of concentration which produces $50 \%$ inhibition $\left(\mathrm{IC}_{50}\right)$

The designation of IC50 value is based on measuring a decrease in the formation of a metabolite in regards to different concentrations of potential inhibitor (studied compounds). In the case of estimating CYP3A4 $\mathrm{IC}_{50}$ values, the monitored reaction is $6 \beta$-hydroxylation of testosterone, and monitored metabolite is $6 \beta$-hydroxytestosterone. Studied compounds were dissolved in dimethylsulfoxide and diluted, the same way as with the incubation procedure of determining leading CYP. Final incubation mixes were comprised of $5 \mu \mathrm{M}$ of testosterone (being an isoform-specific substrate for monitored reaction), human liver microsomes suspension ( 0.5 $\mathrm{mg} / \mathrm{mL})$ in phosphate buffer (100 mM, pH 7.4), NADPH (100 mM), and studied compounds in different concentrations: $0 \mu \mathrm{M}$ (control), $0.1 \mu \mathrm{M}, 0.25$ $\mu \mathrm{M}, 1 \mu \mathrm{M}, 2.5 \mu \mathrm{M}, 10 \mu \mathrm{M}, 25 \mu \mathrm{M}$. Incubation mixes differed only by the studied compound concentration, with each concentration being a separate sample in 96-well plate. Before conducting the incubation, all samples were pre-incubated. Pre-incubation mixes (comprised of testosterone, studied compound, NADPH and in phosphate buffer) were preincubated for $15 \mathrm{~min}$ in $37^{\circ} \mathrm{C}$. The reaction was started with the addition of human liver microsomes suspension using a multi-channel pipette to ensure simultaneous start in all wells. Presented mixes were then incubated for $2 \mathrm{~h}$. After the incubation, mixes were transferred to Eppendorf tubes containing an aliquot of ice-cold acetonitrile, which stopped the reaction. Samples were then centrifuged (10 min, $7500 \mathrm{xg}$ ) to separate protein residue from sample solutions. Solutions separated from protein residue were directly analyzed using LC-MS in single ion monitoring mode, set to $6 \beta$-hydroxytestosterone protonated molecule $(\mathrm{m} / \mathrm{z}=305)$. Acquired data were subsequently plotted (amount of formed metabolite vs logarithm of compound concentration), and $\mathrm{IC}_{50}$ value was calculated using an online AAT Bioquest's $\mathrm{IC}_{50}$ calculator. Studied compounds IC50 values, according to Cyprotex Cytochrome P450 inhibition assay, can be divided into three groups,
Compound 1

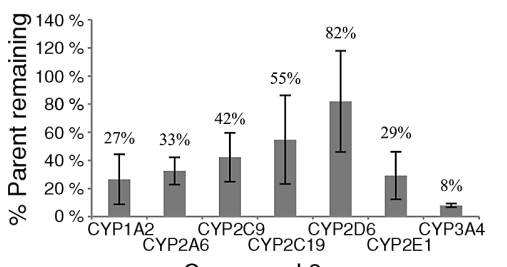

Compound 3

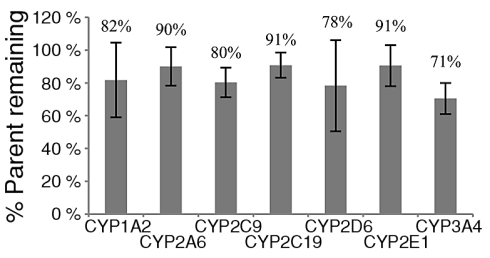

Compound 2

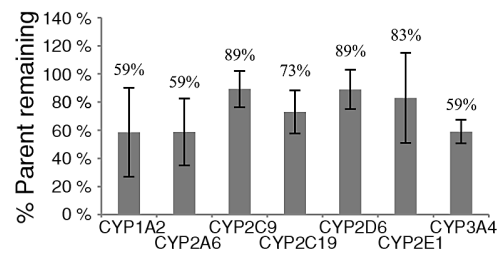

Compound 4

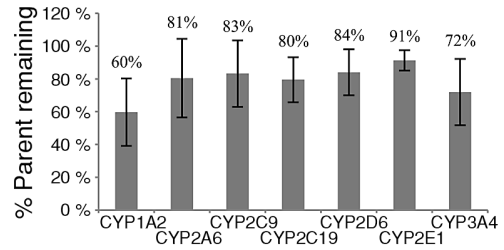

Compound 5

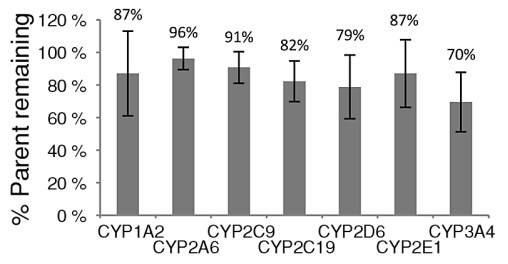

Figure 2. Stability of studied derivatives against baculosomes expressing particular CYP isoenzymes. The error bars represent the standard deviations calculated from all incubations $(n=4)$. 
potent moderate and weak. The threshold for potent inhibition was $\mathrm{IC}_{50}<1 \mu \mathrm{M}$, moderate inhibition was categorized as $\mathrm{IC}_{50}$ between $1 \mu \mathrm{M}$ and $10 \mu \mathrm{M}$ and no or weak inhibition values were $\mathrm{IC}_{50}>10 \mu \mathrm{M}$.

\section{LC-MS assay}

Samples were analyzed using an LC-MS setup consisting of liquid chromatography Agilent 1260 Infinity system with a binary pump, autosampler, temperature-controlled column compartment and UV variable wavelength detector, coupled with an Agilent 6120 Single Quad mass detector and equipped with ESI ion source (Agilent Technologies, USA). Mobile phases consisted of deionized water (phase A) and acetonitrile (phase B), both with $10 \mathrm{mM}$ of ammonium formate $(\mathrm{pH}=3$ ) buffer. Gradient elution program was as follows: 0-6 min $30 \%-80 \%$ B, 6-8 min - 80\%-100\% B, 8-8.10 min $100 \%-30 \%$ B, $8.10-15$ min $30 \%$ B. The flow rate was $0.5 \mathrm{~mL} / \mathrm{min}$. An injection volume of a single sample was $10 \mathrm{~F}$. Samples were separated at $40^{\circ} \mathrm{C}$ on a Poroshell 120 EC-C-18 column $(3 \mathrm{~mm} \times 100$ $\mathrm{mm}, 2.7 \mu \mathrm{m})$, provided by Agilent Technologies. The data were collected and processed by ChemStation software.

Fragmentation and capillary voltage were adjusted using the FIA (Flow Injection Analysis) method. This method allows the injection of a sequence of identical samples, each one being processed under different detector settings. This method allowed for adjusting the fragmentor voltage to $150 \mathrm{~V}$ and capillary voltage to $3000 \mathrm{~V}$. Nitrogen was used as the drying gas, with a flow rate of 10 $\mathrm{L} / \mathrm{min}$ and a drying temperature of $300^{\circ} \mathrm{C}$.

\section{RESULTS}

Identification of CYP isoforms contributing to arylpiperazine drug candidates metabolism

The study focused on 5 selected arylpiperazine derivatives with $5-\mathrm{HT}_{1 \mathrm{~A}}$ receptor inhibitory activity and their interaction with 7 selected cytochrome P450 isoenzymes. All the incubations were per- formed four times. Figure 2 demonstrates the results of all four independent incubations for each of the 5 studied compounds and for each of the 7 studied CYP isoforms. Each graph shows the percentage of the remaining arylpiperazine derivative after the metabolic reaction was carried out by the given isoenzyme (the lower the percentage, the greater the ability of the isoenzyme to metabolize test derivative) in the presence of NADPH after $30 \mathrm{~min}$ of incubation at $37^{\circ} \mathrm{C}$.

All studied CYP isoforms were able to metabolize arylpiperazine derivatives. Figure 2 presents, that after the incubation of compound 1 with $3 \mathrm{~A} 4$, $8 \%$ of the initial concentration of the compound remained in the sample. This means that it was metabolized by the CYP3A4 isoform in 92\%, which was the most rapid biotransformation among all under consideration in this study. CYP3A4 is the most active isoenzyme to metabolize compounds $\mathbf{1}$ $\mathbf{3}, \mathbf{5}$ and also second most potent to metabolize compound 4. The results also show that some of the studied compounds presented rather high metabolic stability (more than $90 \%$ of parent compound remaining) towards CYP isoforms, especially CYP2E1.

All of the studied arylpiperazines are metabolized, to some extent, by isoforms other than CYP3A4. Among test compounds, compound $\mathbf{1}$ is the most susceptible to CYP isoforms and is metabolized by all of the isoforms (with the main contribution from CYP3A4, and with CYP2D6 representing smallest contribution towards metabolism of the studied compound). Compounds 3-5 can be characterized as more metabolically stable (Fig. 2), with compound 4 being metabolized to the slightest degree. Compounds $\mathbf{3}$ and $\mathbf{5}$ stand out among other derivatives, as they are negligibly metabolized by more than one CYP isoform. Compound $\mathbf{3}$ has more than $90 \%$ of initial concentration remaining for CYP2A6, CYP2C19 and CYP2E1, while compound 5 represents a decreased contribution to metabolism by CYP2A6 and CYP2C9 based on the same criteria. Out of 5 studied compounds, 2 represent the

Table 1. Average $\mathrm{IC}_{50}( \pm$ standard deviation, $\mathrm{n}=3$ ) values for all studied compounds, measured for inhibition of testosterone hydroxylation.

\begin{tabular}{|c|c|c|}
\hline Compound & Average $\mathrm{IC}_{50}$ value & Inhibition potency \\
\hline $\mathbf{1}$ & $15.85( \pm 3.79)$ & Weak \\
\hline $\mathbf{2}$ & $6.93( \pm 0.32)$ & Moderate \\
\hline $\mathbf{3}$ & $7.15( \pm 3.82)$ & Moderate \\
\hline $\mathbf{4}$ & $6.13( \pm 4.89)$ & Moderate \\
\hline $\mathbf{5}$ & $8.20( \pm 6.08)$ & Weak/Moderate \\
\hline
\end{tabular}


smallest susceptibility towards CYP2A6 (compounds 3 and 5). Such results suggest that arylpiperazine derivatives can be metabolized by pathways alternative to those involving CYP3A4.

\section{Estimation of $\mathrm{IC}_{50}$ values of studied arylpiper- azine derivatives}

Results from the $\mathrm{IC}_{50}$ estimation study are presented in Table 1. Compounds were categorized using the following assumptions : $\mathrm{IC}_{50}$ value of less than $1 \mu \mathrm{M}$ represent potent inhibitions : values of less than $10 \mu \mathrm{M}$ represents moderate inhibition and values of more than $10 \mu \mathrm{M}$ represent weak inhibition or lack of thereof. Therefore, studied compounds are mostly moderate inhibitors, with the exception of compound 1 being a weak inhibitor of CYP3A4.

\section{DISCUSSION AND CONCLUSION}

Based on the results of this in vitro study, CYP3A4 is primarily responsible for the metabolism of the arylpiperazine derivatives that are part of the presented project. This provides valuable information regarding potential drug-drug interactions arising from arylpiperazine derivatives being used in multi-drug therapy. CYP3A4 is not only the most abundant CYP isoform, but it is also responsible for the metabolism of over $50 \%$ of the marketed drugs (25-27). Therefore, the studied arylpiperazine derivatives might potentially cause drug-drug interactions (leading to worse adverse drug reactions) when co-administered with other CYP3A4 substrates, such as statins (28), erythromycin and clarithromycin (29), nifedipine, amiodarone (20), to name a few. Taking under concern high morbidity of cardiovascular disease, bacterial infections, hypotension, and depressive disorders, co-administration of arylpiperazine derivatives with the abovementioned drugs is a highly probable scenario, especially among elderly patients. One of the most notable CYP3A4 inhibitors is grapefruit juice (recognized as the first drug-food interaction) (30-33), which might also increase the concentration of unchanged arylpiperazine derivative in an organism, leading to adverse drug reactions. Given the fact that the inhibitory effect of grapefruit juice can last up to 37 days after ingestion (34), recognizing CYP3A4 as the main metabolizing isoenzyme for the studied compounds remains of crucial importance for estimation of the safety of those novel drug candidates. St. John's Wort is also a very popular herbal product used in the treatment of depression. As it is not a CYP inhibitor, but an inducer, it is also crucial to mention it when discussing CYP interactions. As St
John's wort increases the rate of CYP3A4 mediated metabolism (35), using it in multi-drug therapy with studied compounds can lead to a decrease of treatment potential, therefore, being a very significant potential drug-drug interaction. Another CYP inducers are antiepileptic drugs such as carbamazepine, phenytoin, phenobarbital and primidone (36-37). Among patients suffering from both depression and epilepsy, those interactions cannot be underestimated, as they rule out co-administration of those two groups of drugs. An aspect that might increase the safety of the studied compounds when used in multidrug therapy is their susceptibility to be metabolized by other CYP isoforms, or - as one can name it alternative metabolic pathways. The capability to be metabolized by different isoforms is very important for the safety of use in multi-drug therapy. If potent CYP3A4 inhibitor is administered, other CYP isoforms can take over its function and conduct the biotransformation regardless of other CYP substrates or inhibitors being present in organism. All tested compounds are metabolized by isoforms other than CYP3A4, albeit to a lesser degree. Compound $\mathbf{1}$ is metabolized by all CYP isoenzymes, which provides multiple alternative metabolic pathways for its metabolism. Additionally, Compound $\mathbf{1}$ average $\mathrm{IC}_{50}$ value of $15.85 \mu \mathrm{M}$ is the highest among all tested compounds. This makes it the safest compound among the tested and set to be co-administered with other drugs. Unfortunately, taking into consideration its metabolic stability value, compound $\mathbf{1}$ turns out to be more susceptible to biotransformation than referent drug, buspirone (13). Therefore, it might prove slightly less valuable, as it might not reach its molecular target when administered and will also be more prone to the first-pass effect. Compound $\mathbf{2}$ is metabolized by CYP3A4 to a smaller degree than compound 1 (59\% of the initial compound concentration is still present in the sample after incubation). However, the same value is designated for both CYP1A2 and CYP2A6, showing similar contributions by those isoforms to metabolism of compound 2. As it is the case for compound $\mathbf{1}$, such property increases safety of the drug when co-administered with other CYP3A4-metabolized drugs, due to the alternative metabolic pathways. The remaining 4 isoforms (CYP2C9, CYP2C19, CYP2D6, and CYP2E1) also contribute to compound 2 biotransformation reactions, but to a smaller degree. The designated $\mathrm{IC}_{50}$ value of $6.93 \mu \mathrm{M}$ places this compound among moderate inhibitors, making it less safe for co-administration than compound $\mathbf{1}$. The described derivative is more resistant to biotransformation reactions than compound $\mathbf{1}$, making it more 
comfortable to administer, yet less safe because of lower $\mathrm{IC}_{50}$ value. Compounds $\mathbf{3}$ and $\mathbf{5}$ stand out among other derivatives because of being metabolized by CYP2A6, CYP2C19, CYP2E1 (for compound 3) and CYP2A6, CYP2C9 (for compound 5) to a very small degree. Such results (more than $90 \%$ of the initial compound concentration left after 30 min incubation) suggest that those isoforms contribute to the metabolism of those specific derivatives negligibly. Therefore, those compounds have fewer alternative metabolic pathways than other candidates from the studied group and therefore may lead to an increased chance of adverse drug reactions in multi-drug therapy. Designated $\mathrm{IC}_{50}$ values of $7.14 \mu \mathrm{M}, 6.13 \mu \mathrm{M}$ and $8.20 \mu \mathrm{M}$ for compounds $\mathbf{3}$, 4 and 5 respectively put them in moderate inhibitors category. Combining those results with not many alternative pathways, those three compounds might prove to be unsafe when co-administered with other CYP3A4 substrates.

As with all the in vitro experiments, the presented study also needs an in vivo confirmation of acquired results. CYP3A4 activity is also regulated by nuclear receptors on the transcriptional level by various molecular mechanisms (such as transactivation and transrepression). Therefore it is crucial to understand, that indirect impact of studied compounds on CYP3A4 activity can only be studied in in vitro cells expressing all the necessary regulatory elements (e.g. hepatocytes or HepRG cell line). Such impact can also be studied during in vivo studies, hence yet again a need for such confirmation, as it is a limiting factor of the presented study. In this study, authors continue their considerations regarding metabolic properties within the set of novel arylpiperazine derivatives with proven activity towards 5-HT receptors. So far, authors assessed their metabolic stability against first phase metabolism using human liver microsomes in in vitro test. Authors also studied metabolic pathways revealing parts of the molecules vulnerable to oxidation by microsomal enzymes (13). In the present paper, authors aimed to further contribute into biochemistry of those derivatives by studying metabolic stability involving particular cytochrome enzymes, identifying leading metabolizing CYP isoform as well as measuring potency to inhibit CYP3A4. All the estimated interactions towards CYP suggest a hazard of adverse drug reactions occurrence, yet whether they are or are not of clinical significance remains unknown. Hence, there is a need to confirm in vitro studies with studies on other models and with clinical trials. However, at the early stage of drug development, such an easy and intuitive assay provides a great amount of initial knowledge about potential drug candidates. Among the studied group, compounds $\mathbf{1}$ and $\mathbf{2}$ can be the best candidate for further studies. Both of those compounds feature a relatively good metabolic stability (even with compound $\mathbf{1}$ being less stable than no. 2) An array of alternative metabolic pathways also increases the safety of usage in multi-drug therapy for both of these candidates. Estimated $\mathrm{IC}_{50}$ values for those compounds put them in two groups, with compound $\mathbf{1}$ being a weak inhibitor (and most likely the safest one to use in multi-drug therapy) and compound $\mathbf{2}$ being a moderate inhibitor (needing an in vitro confirmation of the safety of use in multi-drug therapy). In the current age of patients often having to deal with more than one disease at the same time, much attention should be paid to the issue of drugdrug interactions. Many adverse drug reactions may be even more damaging than symptoms of the disease, and this may be crucial among elderly patients, among which multi-drug therapy is often the case. With depression being not only one of the most prevalent diseases but also one of the most fatal, the need for new therapeutic agents safe for co-administration is vital. This study expands the knowledge in the field of biochemistry of arylpiperazine biochemistry, that can be further used in the drug design to achieve new drug candidates with better properties, especially taking CYP3A4 inhibition as a potential clinical issue.

\section{Conflict of interest}

The authors declare no conflicts of interest.

\section{Funding}

This study was funded by Polish National Science Center (Narodowe Centrum Nauki, https://www. ncn.gov.pl/) (grant number 2015/17/N/ NZ7/04110).

\section{REFERENCES}

1. http://www.who.int/mediacentre/factsheets/ fs369/en/ (accessed on 13.06.2019).

2. Preskorn S.H., Ross R., Stanga C.Y.: Selective Serotonin Reuptake Inhibitors. Antidepressants: Past, Present, and Future. pp. 241-262, Springer, Berlin, Heidelberg 2004.

3. Fournier J.C., DeRubeis R.J., Hollon S.D., Dimidjian S., Amsterdam J.D., et al.: JAMA. 303, 47 (2010).

4. Pies R.: J. Clin. Psychopharmacol. 30, 101 (2010). 
5. Artigas F., Perez V., Alvarez E.: Arch. Gen. Psychiatry. 51, 248 (1994).

6. Salerno L., Pittalà V., Modica M.N., Siracusa M.A., Intagliata S., et al.: Eur. J. Med. Chem. 85C, 716 (2014).

7. Chłoń-Rzepa G., Żmudzki P., Pawłowski M., Wesołowska A., Satała G., et al.: J. Mol. Struct. 1067, 243 (2014).

8. Zygmunt M., Sapa J., Chłoń-Rzepa G., Zagórska A., Siwek A., et al.: Pharmacol. Rep. 66, 505 (2014).

9. Kowalski P., Mitka K., Jaśkowska J., Duszyńska B., Bojarski A.J.: Arch. Pharm. (Weinheim) 346, 339 (2013).

10. Canale V., Guzik P., Kurczab R., Verdie P., Satała G., et al.: Eur. J. Med. Chem. 78, 10 (2014).

11. Chodkowski A., Wróbel M.Z., Turło J., Kleps J., Siwek A., et al.: Eur. J. Med. Chem. 90C, 21 (2014).

12. Ślifirski G., Król M., Kleps J., Ulenberg S., Belka M., et al.: Eur. J. Med. Chem. 166, 144 (2019).

13. Ulenberg S., Belka M., Król M., Herold F., Hewelt-Belka W., et al.: PLoS One. 10, 3 (2015).

14. Zołnowska B., Sławiński J., Pogorzelska A., Szafrański K., Kawiak A., et al.: Molecules 21, 6 (2016).

15. Gomolka A., Ciesielska A., Wróbel M.Z., Chodkowski A., Kleps J., et al.: Eur. J. Med. Chem. 98, 221 (2015).

16. Stefanowicz J., Słowiński T., Wróbel M.Z., Herold F., Gomółka A.E., et al.: Bioorg. Med. Chem. 24, 3994 (2016).

17. Pogorzelska A., Sławiński J., Żołnowska B., Szafrański K., Kawiak A., et al.: Eur J. Med. Chem. 138, 357 (2017).

18. Ulenberg S., Belka M., Baczek T.: J. Chemom. 30, 177 (2016).

19. Tanaka E.: J. Clin. Pharm. Ther. 23, 403 (1998).
20. Lynch T., Price A.: Am. Fam. Physician 76, 391 (2007).

21. Zhu M.S., Zhao W.P., Jimenez H., Zhang D.L., Yeola S., et al.: Drug Metab. Dispos. 33, 500 (2005).

22. Główny Urząd Statystyczny; Stan Zdrowia i Ludności Polski w 2004 r., Warszawa 2006 (in Polish).

23. Andrade C.: J. Clin. Psychiatry 75, 95 (2014).

24. Herold F., Krol M., Kleps J., Nowak G.: Eur. J. Med. Chem. 41, 125 (2006).

25. Zhou S-F.: Curr. Drug Metab. 9, 310 (2008).

26. Kenworthy K.E., Bloomer J.C., Clarke S.E., Houston J.B.: Br. J. Clin. Pharmacol. 48, 716 (1999).

27. Rendic S., Carlo F.J.Di.: Drug Metab. Rev. 29, 413 (1997).

28. Rowan C.G., Brunelli S.M., Munson J., Flory J., Reese P.P., et al.: Pharmacoepidemiol. Drug Saf. 21, 494 (2012).

29. Akiyoshi T., Ito M., Murase S., Miyazaki M., Peter Guengerich F., et al.: Drug Metab. Pharmacokinet. 28, 411 (2013).

30. He K., Iyer K.R., Hayes R.N., Sinz M.W., Woolf T.F., Hollenberg P.F.: Chem. Res. Toxicol. 11, 252 (1998).

31. Bailey D.G., Malcolm J., Arnold O., Spence J.D.: Br. J. Clin. Pharmacol. 46101 (1998).

32. Garg S.K., Kumar N., Bhargava V.K., Prabhakar S.K.: Clin. Pharmacol. Ther. 64, 286 (1998).

33. Bailey D.G., Dresser G.K.: Am. J. Cardiovasc. Drugs. 4, 281 (2004).

34. Lilja J.J., Kivistö K.T., Neuvonen P.J.: Clin. Pharmacol. Ther. 68, 384 (2000).

35. Roby C.A., Anderson G.D., Kantor E., Dryer D.A., Burstein A.H.: Clin. Pharmacol. Ther. 67, 457 (2000).

36. Johannessen S.I., Johannessen Landmark C.: Curr. Neuropharmacol. 8, 254 (2010).

37. Perucca E., Br. J.: Clin. Pharmacol. 61, 246 (2006).

(C) 2020 by Polish Pharmaceutical Society. This is an open access article under the CC BY NC license (c) (1) (9) (http://creativecommons.org/licenses/BY/4.0/). 